\title{
A new set of relativistic screening constants for the screened hydrogenic model
}

\author{
M.A. Mendoza ${ }^{\mathrm{a}, *}$, J.G. Rubiano ${ }^{\mathrm{a}, \mathrm{b}}$, J.M. Gil ${ }^{\mathrm{a}, \mathrm{b}}$, R. Rodríguez ${ }^{\mathrm{a}, \mathrm{b}}$, R. Florido ${ }^{\mathrm{a}, \mathrm{b}}$, P. Martel ${ }^{\mathrm{a}, \mathrm{b}}$, E. Mínguez ${ }^{\mathrm{b}}$ \\ ${ }^{a}$ Departamento de Fisica, Universidad de Las Palmas de Gran Canaria, 35017 Las Palmas de Gran Canaria, Spain \\ ${ }^{\mathrm{b}}$ Instituto de Fusión Nuclear, Universidad Politécnica de Madrid, 28006 Madrid, Spain
}

Keywords:

Screening constants

Screened hydrogenic model

\begin{abstract}
A B S T R A C T
A new Relativistic Screened Hydrogenic Model has been developed to calculate atomic data needed to compute the optical and thermodynamic properties of high energy density plasmas. The model is based on a new set of universal screening constants, including $n l j$-splitting that has been obtained by fitting to a large database of ionization potentials and excitation energies. This database was built with energies compiled from the National Institute of Standards and Technology (NIST) database of experimental atomic energy levels, and energies calculated with the Flexible Atomic Code (FAC). The screening constants have been computed up to the $5 p_{3 / 2}$ subshell using a Genetic Algorithm technique with an objective function designed to minimize both the relative error and the maximum error. To select the best set of screening constants some additional physical criteria has been applied, which are based on the reproduction of the filling order of the shells and on obtaining the best ground state configuration. A statistical error analysis has been performed to test the model, which indicated that approximately $88 \%$ of the data lie within a $\pm 10 \%$ error interval. We validate the model by comparing the results with ionization energies, transition energies, and wave functions computed using sophisticated self-consistent codes and experimental data.
\end{abstract}

\section{Introduction}

The Screened Hydrogenic Model (SHM) is one of the atomic models commonly used in plasma physics. This model determines the energy levels of an electronic configuration as a sum of hydrogenic one-electron energies that are computed with appropriate screened nuclear charges. The SHM used in the average ion model context provides electronic populations with good accuracy and it also allows computing radiative properties $[1-8]$ such as the opacity or the emissivity, as well as thermodynamic properties such as the equation of state or the shock Hugoniot curves [9-11]. For this reason the model has been widely used in the simulation of inertial confinement fusion [12].

In 1930, Slater [13] was the first to introduce the concept of screening constant to calculate the energies of multielectron atoms as a function of the screened nuclear charge, although he only gave empirical rules to estimate these screening constants. Subsequently, Mayer [14] developed a model to computed opacities in plasmas at high density and temperature in which the concept of the average atom was introduced and the SHM was developed and was the first author to compute several sets of universal screening constants.

The theoretical framework of SHM is due to Layzer in the late 1950s [15], who developed a theory based on the concept of screening to determine atomic spectra. In this theory, each atomic state is characterized by a set of screening parameters instead of a wave function. By using a variational principle, a system of algebraic non-linear coupled equations was derived, the solution of which yield the desired screening parameters. However, this theory is not suitable for on-line calculations since the resolution of the equations is quite time consuming and the screening parameters are not universal.

A new SHM based on the ideas of Mayer was calculated using a self-consistent Hartree-Fock-Slater code by More [16], who introduced a new set of screening constants obtained from the fit to a database with 800 ionization potentials of 30 elements. The oneelectron states obtained with this set of screening constants are degenerate with respect to angular momentum, i.e., each state was labeled only by the principal quantum number $n$. To resolve this problem, Perrot [17] applied a first-order perturbation method to the total energy of the More's model to obtain the dependence of the screened charge on the angular quantum number $l$. However, Perrot's model does not provide a new set of screening constants, but a method to correct the screened charges obtained using the 
More's screening constants. Since the More's model was derived in the average atom context, a corrected set of their screening constants, better adapted to a detailed configuration description of the atom, was proposed by Marchand et al. [18].

Faussurier et al. [19] reviewed the theoretical basis of SHM in detail and provided a entire new set of coefficients with $l$-splitting, obtained from the fit to a database with about 15600 values of Hartree-Fock excitation energies and ionization potentials. This model gives more accurate results than those derived by More and Perrot. The original set of coefficients was computed up to the $6 h$ subshell and recently it has been extended up to $10 l$ subshell. The authors also provide theoretical expressions to extend the set of coefficients to $n>10$ [10].

All the above models do not consider the splitting due to relativistic effects. Nevertheless, some authors take them into account using the hydrogenic approximation with relativistic corrections [7,8], and others, use Dirac's relativistic energy expression for the hydrogen atom for the same purpose $[1,20]$. However, currently there does not exist a set of screening constants fit to energy values considering $j$-splitting.

The previous models compute their sets of screening constants using a fit to atomic energies obtained from self-consistent atomic structure codes. In fact, Perrot when reviewing the theoretical basis of the SHM [17], concludes that an empirical adjustment of the screening constants is always necessary to obtain good results, because of the crude approximation of using universal screening constants underlying the SHM model basis in which screening parameters do not depend on each atomic configuration. In spite of this, some authors have developed algorithms for ab initio determination of the screening constants, in both a non-relativistic [21] and in a relativistic framework [22].

There also exist models that do not use screening constants for calculating the screened nuclear charge, such as the model of Nickiforov et al. [23,24], wherein charges are obtained with the trial potential method, using analytical screened hydrogenic wave functions.

In a previous paper $[25,26]$, we developed a method to obtain the screened charges and the external screening of the SHM from analytical potentials. Using this method and analytical screened hydrogenic wave functions, we proposed a SHM with $n l j$-splitting. The model was strictly valid for the ground states of He-like to U-like ions due to the analytical potential used $[27,28]$ and, therefore, it had a limited use for simulation involving excited states, which are important in modeling of NLTE plasmas.

In order to obtain a more flexible atomic model for plasma simulations, in this paper we develop a New Relativistic Screened Hydrogenic Model (NRSHM) based on a new set of universal screening constants obtained by fitting to a database containing a data set of 61,350 high quality atomic data entries. This model has been formulated to treat ground and excited configurations of medium and highly ionized atoms. In Sec. 2, an outline of the model is provided. In Sec. 3 an explanation is given of the structure of the energy level database and the main characteristics of the genetic algorithm (GA) used to fit the screening constants. These are introduced in Sec. 4, together with an statistical error analysis showing that approximately $88 \%$ of the data lie within the $\pm 10 \%$ error interval. Section 5 is devoted to the validation of the NRSHM, which is performed by comparing energy level values, configuration average energies, and wave functions to results computed using more sophisticated models and experimental data.

\section{Description of the model}

As the previous SHM, the NRSHM is based on the independent particle approximation, where each bound electron experiences an effective central potential including the Couloumb interactions with the nucleus and the remaining $N-1$ electrons. The proposed model considers the relativistic $j$-splitting, and thus, a fundamental or excited configuration is written as follows: $\left\{\left(n_{1} l_{1} j_{1}\right)^{P_{1}}, \ldots\right.$,

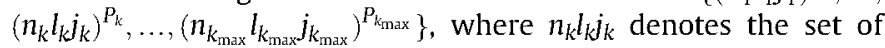
quantum numbers that label the relativistic subshell $k$, and $P_{k}$ is its occupation number which ranges from 0 to $2 j+1$. The average energy $E_{\mathrm{T}}$ of an ion in this configuration is given as the sum of Dirac's eigenvalues for hydrogenic ions:

$$
E_{T}=\sum_{k} P_{k} \epsilon_{k}
$$

$\epsilon_{k}=m_{\mathrm{e}} c^{2}\left[\left(1+\left[\frac{\alpha Q_{k}}{n-j-1 / 2+\sqrt{(j+1 / 2)^{2}-\left(\alpha Q_{k}\right)^{2}}}\right]^{2}\right)^{-1 / 2}-1\right]$

being $m_{\mathrm{e}}$ the electron mass, $c$ the speed of light, $\alpha$ the fine structure constant and $Q_{k}$ the screened nuclear charge experienced by an electron belonging to the $k$ subshell, written as [19]:

$Q_{k}=Z-\sum_{k \prime} \sigma_{k k^{\prime}}\left(P_{k^{\prime}}-\delta_{k k^{\prime}}\right)$

where $Z$ is the nuclear charge, $\delta_{k k^{\prime}}$ is the Kronecker Delta symbol and $\sigma_{k k^{\prime}}$ is the screening constant. The calculation of these universal screening constants is the primary goal of this work.

\section{Description of the fit}

\subsection{Atomic database}

The atomic model is based on a new set of universal screening constants including $j$-splitting, which are obtained from the fit to an extensive database of atomic energies. This database was built with both energies compiled from the NIST database of experimental atomic energy levels [29], and energies calculated with the Flexible Atomic Code (FAC) [30]. The database consists of all the ionization and excitation energy values of isoelectronic series from He to Eu. There are at least 20 different electronic configurations in each isoelectronic series. Each energy value in the database corresponds to both simple excitations and ionization energies, for instance, $1 s_{1 / 2}^{2} 2 s_{1 / 2}^{1} \rightarrow 1 s_{1 / 2}^{2} 4 p_{1 / 2}^{1}$ for Li-like ions. Energies corresponding to the $j$-splitting of a given non-relativistic subshell are also included: $2 p^{2} \rightarrow 2 p_{1 / 2}^{2}, 2 p_{1 / 2}^{1} 2 p_{3 / 2}^{1}, 2 p_{3 / 2}^{2}$, and some energies corresponding to inner-shell excitations such as: $2 s^{2} 2 p^{3} \rightarrow 2 s^{1} 2 p^{4}$. The total number of energy values is 61,350 corresponding to 944 different electronic configurations. The majority of the chosen configuration correspond closely to a standard filling of the atomic subshells [19]. Actually, the database is larger since it also contains energy values of isoelectronic series between $Z=68$ and $Z=92$. However, in this paper we only present results up to $5 p_{3 / 2}$ subshell.

In the fit we have not used energies belonging to either the neutral atom of each element or the first ionization state because we have found that is not possible to obtain a good fit when these are included, as stated by Faussurier et al. [19]. In this regard, the second ionization state of each isoelectronic series of the lanthanides has also been removed.

\subsection{The fit of the screening constants using a genetic algorithm}

For the fit of the screening constants we have designed a procedure based on genetic algorithms (GA), which are stochastic 
Table 1

Table of screening constants $\sigma_{k k^{\prime}}$ of the submatrix $k, k^{\prime}: 1 s_{1 / 2}, 5 p_{3 / 2}$ of the NRSHM.

\begin{tabular}{|c|c|c|c|c|c|c|c|c|c|}
\hline & $1 s_{1 / 2}$ & $2 s_{1 / 2}$ & $2 p_{1 / 2}$ & $2 p_{3 / 2}$ & $3 s_{1 / 2}$ & $3 p_{1 / 2}$ & $3 p_{3 / 2}$ & $3 d_{3 / 2}$ & $3 d_{5 / 2}$ \\
\hline $1 s_{1 / 2}$ & 0.310000 & 0.026843 & 0.004874 & 0.004639 & 0.000000 & 0.000000 & 0.000000 & 0.000000 & 0.000000 \\
\hline $2 s_{1 / 2}$ & 0.687354 & 0.276083 & 0.273158 & 0.266339 & 0.059838 & 0.013608 & 0.013521 & 0.001290 & 0.001125 \\
\hline $2 p_{1 / 2}$ & 0.922154 & 0.326326 & 0.346548 & 0.325740 & 0.071461 & 0.023271 & 0.022907 & 0.006089 & 0.002486 \\
\hline $2 p_{3 / 2}$ & 0.931159 & 0.326403 & 0.366836 & 0.346801 & 0.090301 & 0.047865 & 0.040885 & 0.008301 & 0.007629 \\
\hline $3 s_{1 / 2}$ & 0.931163 & 0.577444 & 0.565396 & 0.565113 & 0.274537 & 0.274040 & 0.253588 & 0.175193 & 0.174976 \\
\hline $3 p_{1 / 2}$ & 0.944595 & 0.802643 & 0.744282 & 0.732800 & 0.286027 & 0.313099 & 0.266505 & 0.313889 & 0.313641 \\
\hline $3 p_{3 / 2}$ & 0.946205 & 0.857747 & 0.746627 & 0.739522 & 0.291605 & 0.358014 & 0.311961 & 0.329175 & 0.321752 \\
\hline $3 d_{3 / 2}$ & 0.991804 & 0.986343 & 0.943187 & 0.935563 & 0.482186 & 0.316150 & 0.314371 & 0.374963 & 0.371989 \\
\hline $3 d_{5 / 2}$ & 0.995148 & 0.991469 & 0.945305 & 0.937540 & 0.487336 & 0.330501 & 0.317032 & 0.385433 & 0.376506 \\
\hline $4 s_{1 / 2}$ & 0.946211 & 0.809161 & 0.776381 & 0.728220 & 0.573596 & 0.573582 & 0.501607 & 0.625283 & 0.600557 \\
\hline $4 p_{1 / 2}$ & 0.960713 & 0.909645 & 0.898199 & 0.821485 & 0.677496 & 0.660517 & 0.632320 & 0.651146 & 0.636113 \\
\hline $4 p_{3 / 2}$ & 0.961982 & 0.923262 & 0.903373 & 0.823412 & 0.704188 & 0.721692 & 0.661577 & 0.695897 & 0.679703 \\
\hline $4 d_{3 / 2}$ & 0.983307 & 0.970650 & 0.966158 & 0.960033 & 0.948710 & 0.922373 & 0.898505 & 0.725244 & 0.701234 \\
\hline $4 d_{5 / 2}$ & 0.987484 & 0.979269 & 0.975108 & 0.974309 & 0.959582 & 0.927196 & 0.906760 & 0.727377 & 0.702986 \\
\hline $4 f_{5 / 2}$ & 0.995662 & 0.995787 & 0.996189 & 0.992692 & 0.945862 & 0.938952 & 0.931108 & 0.932405 & 0.937586 \\
\hline $4 f_{7 / 2}$ & 0.998476 & 0.999104 & 0.998971 & 0.999341 & 0.964455 & 0.956705 & 0.948302 & 0.934684 & 0.960703 \\
\hline $5 s_{1 / 2}$ & 0.965527 & 0.953852 & 0.892634 & 0.845963 & 0.836598 & 0.712495 & 0.662736 & 0.688440 & 0.635962 \\
\hline $5 p_{1 / 2}$ & 0.992639 & 0.987705 & 0.881411 & 0.872450 & 0.853008 & 0.752635 & 0.818444 & 0.764568 & 0.725378 \\
\hline $5 p_{3 / 2}$ & 0.996253 & 0.989251 & 0.964836 & 0.880491 & 0.862306 & 0.838666 & 0.852089 & 0.797661 & 0.728924 \\
\hline
\end{tabular}

numerical optimization algorithms inspired by the theory of evolution. GA's have an advantage over other procedures by performing a greater sweep of the subspace of valid solutions.

In addition, the GA convergence and final result are only weakly dependent on the initial condition as long as it is chosen randomly and the range of variation is sufficiently broad. They also perform a robust and parametric search, i.e., the convergence is guaranteed, provided that the choice of algorithm parameters is appropriate [31]. Since the GA's are efficient in cases where the search is long and complex, and when the solution domain is extensive, they are particularly suitable for obtaining the coefficients of the relativistic hydrogenic screening model. In this case the search space complexity arises from the wide range of atomic data. The GA is initialized starting from the screening constants of Faussurier et al. [19]. Since this set of coefficients does not distinguish the orbitals with different $j$ quantum number, we use the same value for $j$-splitted orbitals. In a first approximation these values are allowed to vary between $\pm 10 \%$, i.e., a range of variation of $\pm 10^{-1}$ for each screening constant has been established as the new search space. After approximately 100 generations, the convergence is achieved, and a new set of coefficients is obtained. The GA is now initialized from this new set and the range of variation is decreased by an order of magnitude. This process is repeated until the range is $\pm 10^{-6}$. The GA is a binary encoded GA with a length of 20 bits per parameter, which produces an accuracy better than one part in a million [31]. The selection of individuals in each generation is made with an operator called "roulette wheel", which ensures that the larger the objective function value the greater the likelihood that the individual passes to the next generation. As crossover operator, a basic crossover was implemented with a probability $P_{\mathrm{c}}=0.60$, so that $60 \%$ of the new population in each generation is formed by applying selection and crossover, and the rest only by selection. The mutation operator was set to a rate of $1 / 20$ by bit and by generation. Elitism was also used, which allows the best individual in each generation to be copied to the next. Since the GA is designed to maximize a fitness function, in order to tackle an error minimization problem, the objective function must be in the following form [32]:

$f_{\text {obj }}=r_{\text {max }}-r(i, t)$

where $r_{\max }$ is a properly selected upper value. Several expressions for $r(i, t)$, all related to some expression of the relative error, were tested and their results compared. They are briefly described below:

\section{Sum of Relative Errors}

$f_{\text {obj }}=r_{\max }-\sum_{i=1}^{N} \epsilon_{\mathrm{r}}(i)=r_{\max }-\sum_{i=1}^{N} \frac{\left|E_{\exp }(i)-E_{\bmod }(i)\right|}{E_{\exp }(i)}$

where $E_{\exp }$ represents the experimental value of the energy and $E_{\text {mod }}$ is the value obtained from the model.

\section{Relative Error with Selection Range}

This function explores the idea of selection range and accuracy. The selection range $R$ is an upper limit, that is, the values higher than $R$ do not contribute to the fit, and at the same time, it is used as a measure of the accuracy of the values.

$f_{\text {obj }}=r_{\max }-\sum_{i=1}^{N} \epsilon_{\mathrm{r}}(R)=r_{\max }-\left|\sum_{i=1}^{N}\left(R-\frac{\left|E_{\exp }(i)-E_{\bmod }(i)\right|}{E_{\exp }(i)}\right)\right|$

\section{Relative Mean Square Error}

The expression for this error is:

$f_{\text {obj }}=r_{\max }-$ RMSE $=r_{\max }-\sqrt{\frac{1}{N} \sum_{i=1}^{N}\left[\frac{E_{\exp }(i)-E_{\bmod }(i)}{E_{\exp }(i)}\right]^{2}}$

$N$ being the total number of experimental data points.

\section{Relative Errors and Maximum Relative Error}

This function is a combination of the sum of relative errors and the maximum relative error value [33].

$f_{\mathrm{obj}}=r_{\mathrm{max}}-\left(\alpha \sum_{i=1}^{N} \epsilon_{\mathrm{r}}(i)+\beta \epsilon_{\mathrm{r}}^{\max }\right)$

$\alpha=1-\beta \quad \beta=1-\frac{\sum_{i=1}^{N} \epsilon_{\mathrm{r}}(i)}{\epsilon_{\mathrm{r}}^{\max }}$

We have finally chosen the last objective function for obtain the acceptability of the individuals in a population because it yields better results. This function is a simple form of a multiobjective function that directly acts on the worst result of each set of individuals, and at the same time minimize the total sum of errors. 
Table 2

Table of screening constants $\sigma_{k k^{\prime}}$ of the submatrix $k, k^{\prime}: 1 s_{1 / 2}, 5 p_{3 / 2}$ of the NRSHM.

\begin{tabular}{|c|c|c|c|c|c|c|c|c|c|c|}
\hline & $4 s_{1 / 2}$ & $4 p_{1 / 2}$ & $4 p_{3 / 2}$ & $4 d_{3 / 2}$ & $4 d_{5 / 2}$ & $4 f_{5 / 2}$ & $4 f_{7 / 2}$ & $5 s_{1 / 2}$ & $5 p_{1 / 2}$ & $5 p_{3 / 2}$ \\
\hline $1 s_{1 / 2}$ & 0.000000 & 0.000000 & 0.000000 & 0.000000 & 0.000000 & 0.000000 & 0.000000 & 0.000000 & 0.000000 & 0.000000 \\
\hline $2 s_{1 / 2}$ & 0.006777 & 0.000433 & 0.000226 & 0.000701 & 0.000612 & 0.000000 & 0.000000 & 0.000000 & 0.000000 & 0.000000 \\
\hline $2 p_{1 / 2}$ & 0.021477 & 0.009807 & 0.009548 & 0.001396 & 0.001239 & 0.000000 & 0.000000 & 0.000000 & 0.000000 & 0.000000 \\
\hline $2 p_{3 / 2}$ & 0.023537 & 0.015806 & 0.012559 & 0.002596 & 0.002225 & 0.000000 & 0.000000 & 0.000000 & 0.000000 & 0.000000 \\
\hline $3 s_{1 / 2}$ & 0.071835 & 0.032258 & 0.028466 & 0.003119 & 0.002735 & 0.000384 & 0.000198 & 0.010507 & 0.003836 & 0.002354 \\
\hline $3 p_{1 / 2}$ & 0.079570 & 0.035987 & 0.023966 & 0.020664 & 0.016675 & 0.001529 & 0.001021 & 0.019019 & 0.011431 & 0.010722 \\
\hline $3 p_{3 / 2}$ & 0.091570 & 0.039760 & 0.028123 & 0.022551 & 0.021412 & 0.003176 & 0.002736 & 0.025215 & 0.032829 & 0.016105 \\
\hline $3 d_{3 / 2}$ & 0.092568 & 0.085482 & 0.061081 & 0.041642 & 0.032507 & 0.024368 & 0.022975 & 0.045098 & 0.020546 & 0.013162 \\
\hline $3 d_{5 / 2}$ & 0.096647 & 0.093655 & 0.085402 & 0.044772 & 0.043081 & 0.024540 & 0.018844 & 0.080244 & 0.047496 & 0.043044 \\
\hline $4 s_{1 / 2}$ & 0.301893 & 0.217471 & 0.198109 & 0.193773 & 0.182372 & 0.120176 & 0.116722 & 0.053658 & 0.035404 & 0.018621 \\
\hline $4 p_{1 / 2}$ & 0.302749 & 0.332135 & 0.279383 & 0.270004 & 0.264208 & 0.246791 & 0.246045 & 0.063882 & 0.059108 & 0.044883 \\
\hline $4 p_{3 / 2}$ & 0.311613 & 0.346999 & 0.317451 & 0.273121 & 0.266488 & 0.300605 & 0.288689 & 0.081908 & 0.076144 & 0.070394 \\
\hline $4 d_{3 / 2}$ & 0.447955 & 0.338928 & 0.317198 & 0.340266 & 0.332231 & 0.279393 & 0.273146 & 0.111448 & 0.080698 & 0.053868 \\
\hline $4 d_{5 / 2}$ & 0.463023 & 0.348033 & 0.334389 & 0.354915 & 0.341973 & 0.286698 & 0.227972 & 0.192517 & 0.125445 & 0.103158 \\
\hline $4 f_{5 / 2}$ & 0.568698 & 0.425590 & 0.401580 & 0.401854 & 0.394334 & 0.387441 & 0.374142 & 0.113721 & 0.086975 & 0.055952 \\
\hline $4 f_{7 / 2}$ & 0.571245 & 0.456563 & 0.427646 & 0.402381 & 0.433531 & 0.409086 & 0.386863 & 0.235591 & 0.102287 & 0.071251 \\
\hline $5 s_{1 / 2}$ & 0.490626 & 0.598787 & 0.567349 & 0.553822 & 0.435666 & 0.719524 & 0.637089 & 0.298852 & 0.336598 & 0.318967 \\
\hline $5 p_{1 / 2}$ & 0.507460 & 0.582875 & 0.603455 & 0.621961 & 0.565777 & 0.728266 & 0.661506 & 0.206857 & 0.325067 & 0.235274 \\
\hline $5 p_{3 / 2}$ & 0.514170 & 0.658522 & 0.639552 & 0.673511 & 0.607985 & 0.734385 & 0.635252 & 0.243956 & 0.401179 & 0.318171 \\
\hline
\end{tabular}

Owing to the fact that the GA can yield good mathematical solutions with no a physical meaning, we introduced a method to manage constraints in our GA. We implemented the method proposed in Ref. [34] that does not need a penalization parameter, using a tournament selection operator where two solutions at a time are compared and the following selection criteria are always enforced:

1. Any feasible solution is preferred to any unfeasible solution.

2. Among two feasible solutions, the one having better objective function value is preferred.

3. Among two unfeasible solutions, the one having smaller constraint violation is preferred.

The fitness function used by this method is:

$f_{\mathrm{a}}=\left\{\begin{array}{cc}f_{\mathrm{obj}} & \text { if } g \geq 0, \quad \forall j=1,2, \ldots, m \\ f_{\max }+\sum_{j=1}^{m}\left\langle g_{j}\right\rangle & \text { otherwise },\end{array}\right.$

where unfeasible solutions are compared based on only their constraint violation. The parameter $f_{\max }$ is the objective function value of the worst feasible solution in the population and $g_{j}$ is the set of constraints [34].

\section{New set of relativistic screened constants}

The set of relativistic screening constants $\left(\sigma_{k k^{\prime}}\right)$ of the submatrix $\left\{k, k^{\prime}: 1 s_{1 / 2}, 5 p_{3 / 2}\right\}$ appears in Tables 1 and 2 . The fit was carried out subshell after subshell, therefore, the acceptability of a group of coefficients depends on the acceptability of the previous ones. In order to minimize error accumulations a set of statistical and physical criteria was imposed for choosing the best set of coefficients to go forward. They are as follows:

Statistical Selection Criteria: a) Distribution of relative errors must have a peaked structure centered around the origin; b) the mean error and the root mean square error must be as small as possible; c) the maximum relative error value must be as small as possible [19]; and d) the number of relative error values within the interval $[-1 \%,+1 \%]$ must be as large as possible.

Physical Selection Criteria: a) The values of coefficients corresponding to levels with $j$-splitting cannot differ by more than a specified limit; b) Since coefficients in a row represent the screening of the outer electrons over the internal ones, their values have to vanish when moving towards the right in a row of the table. On the other hand, coefficients in a column represent the internal screening, that is, the screening of an external electron due to internal electrons, so their values have to increase up to 1 when moving towards the bottom in a column. c) An acceptable set of coefficients has to yield the minimum energy value for the ground configuration of an ion [19].

Fig. 1 presents relative error distributions of the 61,356 data used for the fit, for the NRSHM, More's model and Faussurier's model. For the two latter models, we computed the energies by substituting in Eq. (2) the screened charges that these models provide, instead of using the non-relativistic expression. The NRSHM has a distribution with a main sharp structure centered around the origin, containing $44.23 \%$ of errors in the interval $[-1 \%,+1 \%]$ and $88.29 \%$ in the interval $[-10 \%,+10 \%]$. This structure is more symmetrical than those found using Faussurier's or More's model and its wings are also more symmetric and Gaussian-like, due to the fact that the mean error and root mean square error are much lower. Specifically, the mean error is a factor of 3.65 lower than Faussurier's, and the root mean square error is reduced by a factor of 2.08. In relation to the More's model the behaviour is even better.

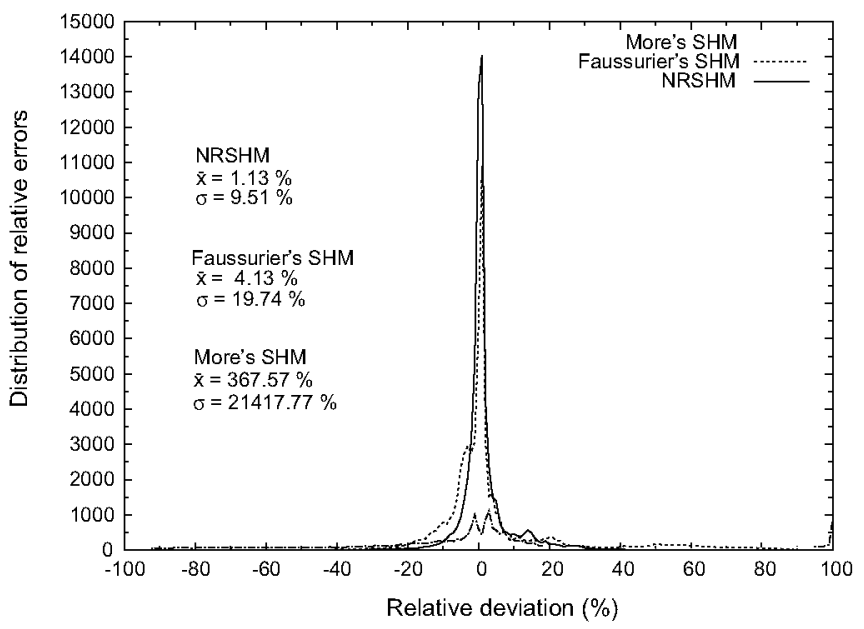

Fig. 1. Relative error distributions of the energy values for the RSHM, More's model and Faussurier's model. $\bar{x} y \sigma$ are respectively the mean and root mean square errors. 


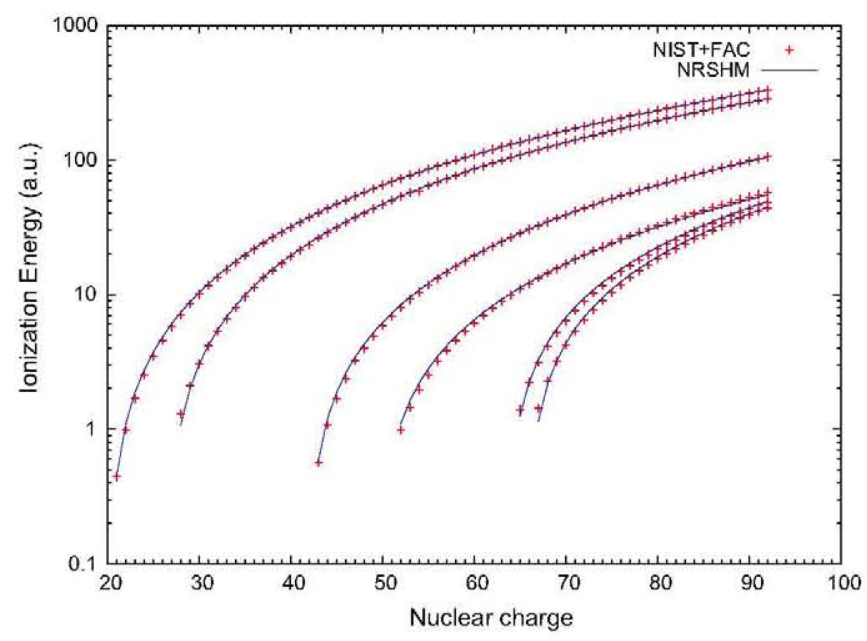

Fig. 2. Comparison of ionization energy values computed by NRSHM (solid line) with NIST and FAC values (+) for $\mathrm{Ca}, \mathrm{Fe}, \mathrm{Mo}, \mathrm{Sn}, \mathrm{Sm}$ and $\mathrm{Gd}$ isolectronic sequences.

In Fig. 2 we illustrate the accuracy of the fit through the comparison of ionization energy values computed by NRSHM with NIST and FAC values for $\mathrm{Ca}, \mathrm{Fe}, \mathrm{Mo}, \mathrm{Sn}, \mathrm{Sm}$ and $\mathrm{Gd}$ isolectronic series. As it can be seen the agreement is very good and it improves for each isolectronic sequence as the nuclear charge $Z$ rises. This is because with increasing degree of ionization, the electron-electron operator diminishes in relation to the electron-nucleus operator, so the Hamiltonian system becomes more hydrogenic and it is better modeled by the screened hydrogenic approximation. For this reason, we consider it necessary to perform an independent fit for the neutral and quasi-neutral species and thus obtain an independent set of screening constants for them. Nevertheless, since the main application of the SHM is to simulate the atomic physic for high energy density plasmas, neutral and quasi-neutral species are not usually involved in the physics of the problem. The same behaviour is shown in Fig. 3 where the different ionization energies of several ions are plotted.

\section{Results}

To check the quality of the model we have compared several atomic quantities calculated with our model, with data obtained by numerical solution of the Dirac equation and with More's and Faussurier's versions of the SHM. We also have compared with proven results both experimental and theoretical from the literature. In the next section we present the results for these comparisons, for configuration averaged energies, electron binding energies, transition energies, and radial wave functions.

\subsection{Configuration average energies}

Table 3 shows comparisons among experimental configuration average energies obtained from NIST [29] with the NRSHM results. The values for moderately and highly ionized ions are very close to the experimental values. As mentioned above the agreement is worse for neutral or quasi-neutral species, and it is clearly confirmed in the case of the neutral iron, where errors around $50 \%$ are obtained. This behaviour depends on the number of electron of the atom because the higher the value of $Z$ the more errors are accumulated in the fit of the constants. Therefore, for a low $Z$ material the results for neutral are better, as shown in Table 4, where experimental values of the configuration average energy for the ground state of several ionization stages of nitrogen and oxygen ions [35] are compared with the ones obtained by different models: Hartree-Fock (HF) [36], Hartree-Fock-Slater (HFS) [37], DFT (D) [38], and the NRSHM.
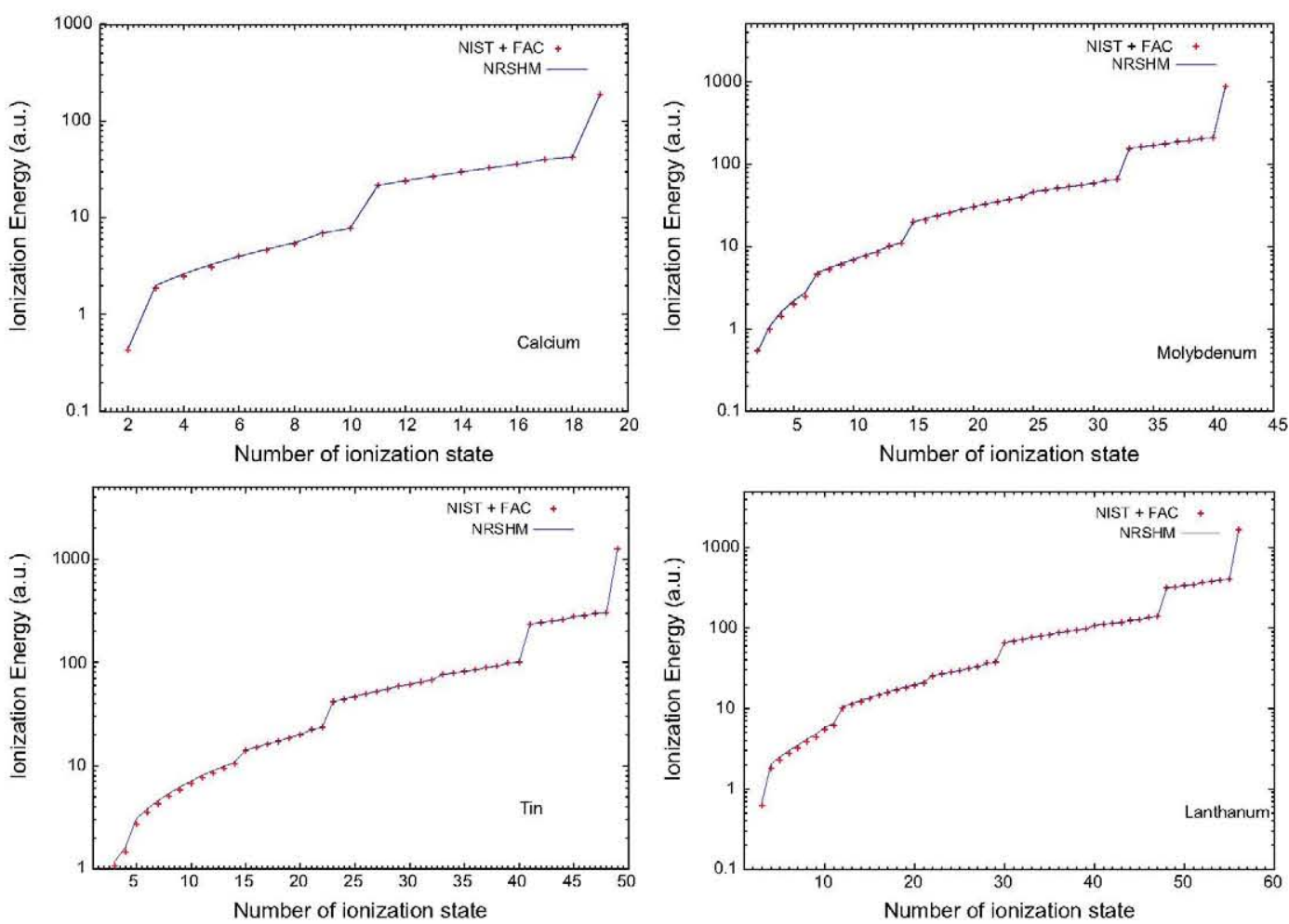

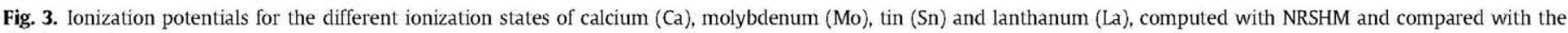
database values. 
Table 3

Comparison of experimental ionization energies from NIST with values computed with NRSHM.

\begin{tabular}{|c|c|c|c|c|c|c|c|c|c|c|c|c|c|c|c|}
\hline \multirow[t]{2}{*}{ Atomic Number } & \multicolumn{3}{|l|}{ C-like } & \multicolumn{3}{|l|}{ Ne-like } & \multicolumn{3}{|l|}{ Al-like } & \multicolumn{3}{|l|}{ Ar-like } & \multicolumn{3}{|l|}{ Fe-like } \\
\hline & Exp & NRSHM & $\epsilon_{\mathrm{r}}(\%)$ & Exp & NRSHM & $\epsilon_{\mathrm{r}}(\%)$ & Exp & NRSHM & $\epsilon_{\mathrm{r}}(\%)$ & Exp & NRSHM & $\epsilon_{\mathrm{r}}(\%)$ & Exp & NRSHM & $\epsilon_{\mathrm{r}}(\%)$ \\
\hline 6 & 0.4138 & 0.3638 & 12.08 & & & & & & & & & & & & \\
\hline 7 & 1.3484 & 1.0450 & 22.51 & & & & & & & & & & & & \\
\hline 8 & 2.3443 & 1.9763 & 15.70 & & & & & & & & & & & & \\
\hline 9 & 3.2023 & 3.1581 & 1.38 & & & & & & & & & & & & \\
\hline 10 & 4.6383 & 4.5905 & 1.03 & 0.7925 & 0.5594 & 29.41 & & & & & & & & & \\
\hline 11 & 6.3276 & 6.2738 & 0.85 & 1.7377 & 1.5454 & 11.07 & & & & & & & & & \\
\hline 12 & 8.2695 & 8.2084 & 0.74 & 2.9452 & 2.7810 & 5.57 & & & & & & & & & \\
\hline 13 & 10.4610 & 10.3946 & 0.63 & 4.4096 & 4.2664 & 3.25 & 0.2200 & 0.1810 & 17.71 & & & & & & \\
\hline 14 & 12.9035 & 12.8330 & 0.55 & 6.1286 & 6.0015 & 2.07 & 0.6007 & 0.6108 & -1.68 & & & & & & \\
\hline 15 & 15.5970 & 15.5239 & 0.47 & 8.1004 & 7.9863 & 1.41 & 1.1099 & 1.1519 & -3.78 & & & & & & \\
\hline 16 & 18.5500 & 18.4680 & 0.44 & 10.3247 & 10.2208 & 1.01 & 1.7354 & 1.8043 & -3.97 & & & & & & \\
\hline 17 & 21.7519 & 21.6657 & 0.40 & 12.7992 & 12.7052 & 0.73 & 2.4923 & 2.5681 & -3.04 & & & & & & \\
\hline 18 & 25.2050 & 25.1179 & 0.35 & 15.5280 & 15.4394 & 0.57 & 3.3445 & 3.4435 & -2.96 & 0.5792 & 0.6332 & -9.33 & & & \\
\hline 19 & 28.9100 & 28.8251 & 0.29 & 18.5100 & 18.4234 & 0.47 & 4.3205 & 4.4307 & -2.55 & 1.1623 & 1.2652 & -8.85 & & & \\
\hline 20 & 32.8750 & 32.7882 & 0.26 & 21.7500 & 21.6575 & 0.43 & 5.4110 & 5.5298 & -2.19 & 1.8710 & 2.0083 & -7.33 & & & \\
\hline 21 & 37.1000 & 37.0079 & 0.25 & 25.2600 & 25.1415 & 0.47 & 6.6150 & 6.7409 & -1.90 & 2.7007 & 2.8623 & -5.99 & & & \\
\hline 22 & 41.5550 & 41.4851 & 0.17 & 28.9525 & 28.8757 & 0.27 & 7.9349 & 8.0643 & -1.63 & 3.6492 & 3.8275 & -4.89 & & & \\
\hline 23 & 46.2924 & 46.2208 & 0.15 & 32.9286 & 32.8601 & 0.21 & 9.3952 & 9.5002 & -1.12 & 4.7085 & 4.9038 & -4.15 & & & \\
\hline 24 & 51.3000 & 51.2159 & 0.16 & 37.1387 & 37.0948 & 0.12 & 10.9550 & 11.0489 & -0.86 & 5.8863 & 6.0912 & -3.48 & & & \\
\hline 25 & 56.5500 & 56.4715 & 0.14 & 41.7000 & 41.5799 & 0.29 & 12.6250 & 12.7105 & -0.68 & 7.1500 & 7.3898 & -3.35 & & & \\
\hline 26 & 62.0573 & 61.9886 & 0.11 & 46.3835 & 46.3156 & 0.15 & 14.4117 & 14.4854 & -0.51 & 8.5841 & 8.7997 & -2.51 & 0.2904 & 0.1616 & 44.37 \\
\hline 27 & 67.8500 & 67.7684 & 0.12 & 51.3450 & 51.3020 & 0.08 & 16.3000 & 16.3739 & -0.45 & 10.1200 & 10.3209 & -1.99 & 0.6278 & 0.2609 & 58.44 \\
\hline 28 & 73.9000 & 73.8122 & 0.12 & 56.6500 & 56.5392 & 0.20 & 18.3000 & 18.3763 & -0.42 & 11.7950 & 11.9535 & -1.34 & 1.2931 & 1.0733 & 17.00 \\
\hline 29 & 80.1915 & 80.1212 & 0.09 & 62.1029 & 62.0273 & 0.12 & 20.4716 & 20.4928 & -0.10 & 13.5551 & 13.6975 & -1.05 & 2.1087 & 1.9964 & 5.32 \\
\hline 30 & 86.7526 & 86.6968 & 0.06 & 67.8438 & 67.7666 & 0.11 & 22.7361 & 22.7240 & 0.05 & 15.4232 & 15.5530 & -0.84 & 3.0345 & 3.0302 & 0.14 \\
\hline 31 & 93.0404 & 93.5404 & -0.54 & 73.8354 & 73.7571 & 0.11 & 25.0507 & 25.0701 & -0.08 & 17.3186 & 17.5201 & -1.16 & 4.1417 & 4.1747 & -0.80 \\
\hline 32 & 100.8317 & 100.6534 & 0.18 & 80.0001 & 79.9992 & 0.00 & 27.4747 & 27.5315 & -0.21 & 19.5467 & 19.5989 & -0.27 & 5.3264 & 5.4298 & -1.94 \\
\hline
\end{tabular}

In Table 5 the total energies for several Na-like ions estimated from the current model are compared with the Extended HartreeFock calculations of Fraga et al. [39], the DFT DAVID code [38], and with the SHM using the screening constants of More and Faussurier. Again the NRSHM model provides a good agreement for moderately and highly ionized ions providing a relative error in relation to the DAVID results that is lower than the $0.25 \%$ in the worst case. We note the good accuracy obtained for heavy elements, when the relativistic effects become important, in relation to the accuracy of the nonrelativistc SHM of More and Faussurier et al.

\subsection{Electron binding energies}

Although the fundamental magnitude when describing multielectron atoms is the configuration average energy of one electronic configuration, frequently, the electron binding energies are used as fundamental parameters for calculation of electronic transition energies or oscillator strengths. Faussurier et al. [19] give

Table 4

Configuration average energy for the ground state of several ionization stages of nitrogen and oxygen ions. Experimental data (Exp), NRSHM, Hartree-Fock (HF), Hartree-Fock-Slater (HFS) and Density Functional Theory (D).

\begin{tabular}{lllllll}
\hline & Configuration & $E_{\mathrm{T}}^{\mathrm{EXP}}$ & $E_{\mathrm{T}}^{\mathrm{NRSHM}}$ & $E_{\mathrm{T}}^{\mathrm{HF}}$ & $E_{\mathrm{T}}^{\mathrm{HFS}}$ & $E_{\mathrm{T}}^{\mathrm{D}}$ \\
\hline $\mathrm{N}-\mathrm{I}$ & $1 s_{1 / 2}^{2} 2 s_{1 / 2}^{2} 2 p_{1 / 2}^{2} 2 p_{3 / 2}^{1}$ & -54.80 & -54.45 & -54.30 & -53.56 & -53.60 \\
$\mathrm{~N}-\mathrm{II}$ & $1 s_{1 / 2}^{2} 2 s_{1 / 2}^{2} 2 p_{1 / 2}^{2}$ & -54.45 & -54.03 & -53.85 & -53.12 & -53.15 \\
$\mathrm{~N}-\mathrm{III}$ & $1 s_{1 / 2}^{2} 2 s_{1 / 2}^{2} 2 p_{1 / 2}^{1}$ & -52.99 & -52.98 & -52.82 & -52.13 & -52.14 \\
$\mathrm{~N}-\mathrm{IV}$ & $1 s_{1 / 2}^{2} 2 s_{1 / 2}^{2}$ & -51.25 & -51.22 & -51.08 & -50.47 & -50.46 \\
$\mathrm{~N}-\mathrm{V}$ & $1 s_{1 / 2}^{2} 2 s_{1 / 2}^{1}$ & -48.40 & -48.38 & -48.33 & -47.75 & -47.67 \\
$\mathrm{~N}-\mathrm{VI}$ & $1 s_{1 / 2}^{2}$ & -44.80 & -44.78 & -44.74 & - & -44.18 \\
$\mathrm{O}-\mathrm{I}$ & $1 s_{1 / 2}^{2} 2 s_{1 / 2}^{2} 2 p_{1 / 2}^{2} 2 p_{3 / 2}^{2}$ & -75.08 & -74.94 & -74.77 & -73.91 & -73.98 \\
$\mathrm{O}-\mathrm{II}$ & $1 s_{1 / 2}^{2} 2 s_{1 / 2}^{2} 2 p_{1 / 2}^{2} 2 p_{3 / 2}^{1}$ & -74.50 & -74.46 & -74.24 & -73.37 & -73.43 \\
$\mathrm{O}-\mathrm{III}$ & $1 s_{1 / 2}^{2} 2 s_{1 / 2}^{2} 2 p_{1 / 2}^{2}$ & -73.28 & -73.28 & -73.05 & -72.21 & -72.25 \\
$\mathrm{O}-\mathrm{IV}$ & $1 s_{1 / 2}^{2} 2 s_{1 / 2}^{2} 2 p_{1 / 2}^{1}$ & -71.30 & -71.31 & -71.1 & -70.3 & -70.32 \\
$\mathrm{O}-\mathrm{V}$ & $1 s_{1 / 2}^{2} 2 s_{1 / 2}^{2}$ & -68.46 & -68.44 & -68.26 & -67.56 & -67.56 \\
$\mathrm{O}-\mathrm{Vl}$ & $1 s_{1 / 2}^{2} 2 s_{1 / 2}^{1}$ & -64.27 & -64.26 & -64.18 & -63.51 & -63.44 \\
$\mathrm{O}-\mathrm{VII}$ & $1 s_{1 / 2}^{2}$ & -59.20 & -59.18 & -59.11 & - & -58.49 \\
\hline
\end{tabular}

an extensive discussion of the physical meaning of individual electron binding energies, and explain three different methods for calculating them, namely, the $X \alpha$ model, the Hartree-Fock model and derivation in $1 / 2$ states. In the $X \alpha$ model $[13,40]$, the electron binding energies are calculated by derivation of the total energy, $E_{\mathrm{T}}$ with respect to the population $P_{k}$ The Hartree-Fock model obtains the binding energies by averaging the kinetic energy, the potential energies of interaction with the nucleus, and the electrostatic interactions between the electrons using screened hydrogenic wave functions. Finally, derivation in $1 / 2$ states assumes that the energy of a transition $k_{0} \rightarrow k_{0}^{\prime}$ is equal to the difference between the electron binding energies $\epsilon_{k_{0}}^{X \alpha}$ and $\epsilon_{k_{0}^{\prime}}^{X \alpha}$ when these quantities are evaluated with the configuration $\left(P_{1}, \ldots, P_{k_{0}}-1 / 2, \ldots, P_{k_{0}^{\prime}}+1 / 2, \ldots\right.$, $\left.P_{k \max }\right)$. In the same way, $\epsilon_{k_{0}}^{X \alpha}$ is equal to the ionization potential if the derivation is performed with $\left(P_{1}, \ldots, P_{k_{0}}-1 / 2, \ldots, P_{k \max }\right)$ [19].

Table 6 shows electron binding energies for several Ne-like ions computed with the three approximations and using relativistic

Table 5

Configuration average energy for several Na-like ions computed with different codes: DFT David (D), Hartree-Fock (HF), Rubiano et al. (RH), this model (NRSHM), More (M) and Faussurier et al. (F).

\begin{tabular}{lrllrrr}
\hline $\begin{array}{l}\text { Atomic } \\
\text { Number }\end{array}$ & $E_{\mathrm{D}}$ & \multicolumn{1}{l}{$E_{\mathrm{HF}}$} & \multicolumn{1}{l}{$E_{\mathrm{RH}}$} & \multicolumn{1}{l}{$E_{\mathrm{NRSHM}}$} & \multicolumn{2}{l}{$E_{\mathrm{M}}$} \\
\hline 18 & & & & & & $E_{\mathrm{F}}$ \\
20 & -512.1 & -512.9 & -511.0 & -513.3 & -504.8 & -511.3 \\
23 & -649.8 & -650.8 & -648.7 & -651.2 & -640.4 & -648.1 \\
26 & -888.0 & -889.2 & -886.6 & -889.7 & -874.8 & -884.1 \\
30 & -1164.5 & -1165.9 & -1162.9 & -1166.4 & -1146.2 & -1157.1 \\
34 & -1593.3 & -1594.7 & -1591.4 & -1595.3 & -1565.5 & -1578.6 \\
36 & -2091.9 & -2093.0 & -2089.5 & -2093.9 & -2050.7 & -2066.0 \\
47 & -2367.6 & -2368.6 & -2365.1 & -2369.7 & -2317.9 & -2334.3 \\
50 & -4210.9 & - & -4206.9 & -4211.7 & -4081.7 & -4104.1 \\
54 & -4812.8 & - & -4808.2 & -4812.8 & -4649.1 & -4673.1 \\
55 & -5684.2 & - & -5678.8 & -5683.0 & -5463.1 & -5489.3 \\
67 & -5914.5 & - & -5909.1 & -5913.1 & -5676.9 & -5703.6 \\
79 & -9094.9 & - & -9086.3 & -9086.5 & -8563.0 & -8596.3 \\
\hline
\end{tabular}


Table 6

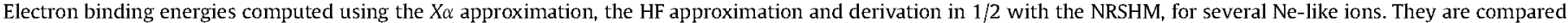
with values from FAC, GRASP, DFT David code and Smith's SHM model.

\begin{tabular}{|c|c|c|c|c|c|c|c|c|}
\hline Atomic Number & $n l j$ & $-\epsilon_{\mathrm{FAC}}$ & $-\epsilon_{\text {GRASP }}$ & $-\epsilon_{\mathrm{D}}$ & $-\epsilon_{\text {Smith }}$ & $X \alpha$ & $-\epsilon_{\text {NRSHM }} H F$ & der $1 / 2$ \\
\hline \multirow[t]{4}{*}{16} & $1 s_{1 / 2}$ & 94.52 & 95.46 & 92.43 & 95.66 & 91.66 & 96.53 & 92.65 \\
\hline & $2 s_{1 / 2}$ & 11.74 & 12.65 & 11.87 & 12.65 & 11.96 & 12.84 & 13.84 \\
\hline & $2 p_{1 / 2}$ & 9.78 & 10.32 & 9.92 & 10.19 & 9.34 & 10.37 & 11.68 \\
\hline & $2 p_{3 / 2}$ & 9.73 & 10.27 & 9.88 & 10.15 & 9.29 & 10.31 & 11.70 \\
\hline \multirow[t]{4}{*}{26} & $1 s_{1 / 2}$ & 280.64 & 283.08 & 277.58 & 283.46 & 276.17 & 284.25 & 274.21 \\
\hline & $2 s_{1 / 2}$ & 49.58 & 51.13 & 49.80 & 51.19 & 49.86 & 51.45 & 48.91 \\
\hline & $2 p_{1 / 2}$ & 45.63 & 46.66 & 45.92 & 46.67 & 44.93 & 46.84 & 44.43 \\
\hline & $2 p_{3 / 2}$ & 45.15 & 46.18 & 45.44 & 46.24 & 44.52 & 46.41 & 44.43 \\
\hline \multirow[t]{4}{*}{66} & $1 s_{1 / 2}$ & 2143.41 & 2153.60 & 2136.90 & 2151.44 & 2128.97 & 2152.13 & 2139.7 \\
\hline & $2 s_{1 / 2}$ & 485.01 & 490.14 & 485.67 & 485.39 & 481.99 & 486.71 & 494.98 \\
\hline & $2 p_{1 / 2}$ & 471.67 & 475.23 & 472.34 & 472.18 & 466.20 & 472.03 & 482.68 \\
\hline & $2 p_{3 / 2}$ & 440.87 & 444.62 & 441.76 & 443.07 & 438.28 & 443.78 & 453.26 \\
\hline \multirow[t]{4}{*}{91} & $1 s_{1 / 2}$ & 4457.02 & 4429.20 & 4455.50 & 4470.40 & 4434.03 & 4471.47 & 4451.87 \\
\hline & $2 s_{1 / 2}$ & 1062.42 & 1063.30 & 1064.60 & 1053.00 & 1049.37 & 1056.65 & 1071.31 \\
\hline & $2 p_{1 / 2}$ & 1041.33 & 1036.10 & 1041.80 & 1033.50 & 1023.35 & 1032.36 & 1051.16 \\
\hline & $2 p_{3 / 2}$ & 902.80 & 900.76 & 904.05 & 901.90 & 896.20 & 904.13 & 917.81 \\
\hline
\end{tabular}

expressions (see appendices). We compare our results with the those obtained from FAC [30], GRASP [41], DFT DAVID code [38], and Smith's SHM model [22]. As can be seen, the $X \alpha$ type values are closer to the calculations of DAVID code, while the HF type values agree better with GRASP. Thus, we can see that the differences among the numerical models is similar to that found between the NRSHM and any of them. Nevertheless, we remark that for isolated atoms it is better to compute the transition energies as differences between configuration average energies than between oneelectron binding energies as Faussurier et al. indicate in their paper. The real utility of these parameters is found in the average atom approach for computing the average ionization of plasmas where the one-electron binding energies in the $X \alpha$ approximation are a key piece in the model [10].

Electron binding energies of $\mathrm{Au}^{+25}$ (a.u.) computed with NRSHM using the $X \alpha$ approximation are shown in Table 7. They are compared with values computed with the relativistic semiclassical values of D'yachkov and Pancratov [42], the relativistic SCF calculations of Yabe and Goel [43], Mabong's values (Mb) [44], the DFT DAVID code [38] and Rubiano et al.'s RSHM [25]. As seen, the discrepancies of the NRSHM results with respect to the rest of the models are in the same order of magnitude as the diferences among them. Finally, in Table 8 we show electron binding energies for several iron ions computed with NRSHM using both $X \alpha$ and HF

Table 7

Comparison of $A u^{+25}$ energy levels (a.u.) calculated with the NRSHM and several models: DAVID (D), Yabe and Goel (YG), Mabong (Mb), D'yachkov and Pankaratov (DP), and Rubiano et al. (RH).

\begin{tabular}{lrrrrrr}
\hline$n l j$ & \multicolumn{1}{c}{$-\epsilon_{\mathrm{D}}$} & \multicolumn{1}{c}{$-\epsilon_{\mathrm{YG}}$} & \multicolumn{1}{c}{$-\epsilon_{\mathrm{Mb}}$} & \multicolumn{1}{c}{$-\epsilon_{\mathrm{DP}}$} & \multicolumn{1}{c}{$-\epsilon_{\mathrm{RH}}$} & \multicolumn{1}{c}{$-\epsilon_{\mathrm{NRSHM}}$} \\
\hline $1 s_{1 / 2}$ & 2995.1 & 3005.8 & 2981.7 & 2994.7 & 2960.1 & 2984.4 \\
$2 s_{1 / 2}$ & 556.6 & 555.9 & 556.1 & 540.9 & 555.5 & 542.7 \\
$2 p_{1 / 2}$ & 535.0 & 535.1 & 536.4 & 519.7 & 534.4 & 526.1 \\
$2 p_{3 / 2}$ & 467.8 & 466.9 & 472.6 & 476.1 & 468.9 & 461.9 \\
$3 s_{1 / 2}$ & 158.2 & 153.7 & 153.5 & 149.6 & 158.0 & 149.0 \\
$3 p_{1 / 2}$ & 148.6 & 144.2 & 145.9 & 139.4 & 148.1 & 139.3 \\
$3 p_{3 / 2}$ & 133.7 & 129.0 & 132.1 & 130.7 & 133.8 & 129.9 \\
$3 d_{3 / 2}$ & 117.8 & 113.2 & 115.6 & 115.5 & 118.5 & 112.6 \\
$3 d_{5 / 2}$ & 114.6 & 109.9 & 112.8 & 112.9 & 115.2 & 109.7 \\
$4 s_{1 / 2}$ & 58.3 & 54.5 & 53.0 & 52.9 & 57.1 & 53.7 \\
$4 p_{1 / 2}$ & 54.2 & 50.3 & 49.8 & 48.6 & 52.3 & 48.6 \\
$4 p_{3 / 2}$ & 49.9 & 46.4 & 46.2 & 46.3 & 49.2 & 46.1 \\
$4 d_{3 / 2}$ & 43.3 & 39.6 & 37.8 & 39.7 & 42.3 & 39.1 \\
$4 d_{5 / 2}$ & 42.4 & 38.8 & 37.2 & 39.1 & 41.3 & 38.6 \\
$4 f_{5 / 2}$ & 34.2 & 30.1 & 29.7 & 30.9 & 33.2 & 30.3 \\
$4 f_{7 / 2}$ & 33.9 & 29.9 & 29.6 & 30.7 & 33.0 & 29.7 \\
\hline
\end{tabular}

approximations, and compared with D'yachkov et al.'s model [42], self-consistent-field (Cowan's code) and parametric-potential method (OPAL code) calculations [45], and experimental data interpolation [46]. Results obtained with $X \alpha$ approximation are smaller than experimental values and values from the others models. However, values obtained with HF approximation fit better to experimental values, and, in some cases, they are better than the ones obtained for the other models.

\subsection{Transition energies}

Transition energies play an important role in radiative process in plasmas. Since transition energies have been used to fit the screening constants the ability of the NRSHM to compute these

Table 8

Energy levels of different iron ions (eV), calculated with the NRSHM using both $X \alpha$ and HF approximations, and compared with D'yachkov et al's model, selfconsistent-field (Cowan's code) and parametric-potencial-method (OPAL code) calculations, and experimental data interpolation on results [46].

\begin{tabular}{|c|c|c|c|c|c|c|c|}
\hline $\mathrm{Fe}^{+2}$ & $n l j$ & $-\epsilon_{\mathrm{DP}}$ & $-E_{\text {Cowan }}$ & $-\epsilon_{\text {OPAL }}$ & $-\epsilon_{\text {Lotz }}$ & $-\epsilon_{\mathrm{NRSHM}}^{\mathrm{X} \alpha}$ & $-\mathrm{E}_{\mathrm{NRSHM}}^{\mathrm{HF}}$ \\
\hline \multirow[t]{7}{*}{$3 d^{6}$} & $1 s_{1 / 2}$ & 7238 & 7156 & 7145 & 7210 & 6989.28 & 7209.17 \\
\hline & $2 s_{1 / 2}$ & 860 & 884 & 896 & 913 & 850.70 & 893.45 \\
\hline & $2 p_{1 / 2}$ & 745 & 748 & 771 & 788 & 737.21 & 788.47 \\
\hline & $2 p_{3 / 2}$ & 736 & - & - & 775 & 704.61 & 754.83 \\
\hline & $3 s_{1 / 2}$ & 112 & 130 & 125 & 141 & 127.51 & 141.43 \\
\hline & $3 p_{1 / 2}$ & 76 & 91 & 88 & 105 & 86.48 & 100.01 \\
\hline & $3 p_{3 / 2}$ & 75 & - & - & 103 & 83.62 & 96.59 \\
\hline \multicolumn{8}{|l|}{$\mathrm{Fe}^{+6}$} \\
\hline \multirow[t]{7}{*}{$3 d^{2}$} & $1 s_{1 / 2}$ & 7343 & 7261 & 7272 & 7394 & 7092.70 & 7312.59 \\
\hline & $2 s_{1 / 2}$ & 974 & 992 & 1002 & 1033 & 959.02 & 1001.78 \\
\hline & $2 p_{1 / 2}$ & 857 & 856 & 867 & 905 & 843.66 & 894.96 \\
\hline & $2 p_{3 / 2}$ & 847 & - & - & 902 & 812.33 & 862.62 \\
\hline & $3 s_{1 / 2}$ & 213 & 223 & 216 & 227 & 219.63 & 234.15 \\
\hline & $3 p_{1 / 2}$ & 176 & 180 & 178 & 192 & 176.59 & 191.32 \\
\hline & $3 p_{3 / 2}$ & 175 & - & - & 190 & 173.83 & 188.04 \\
\hline \multicolumn{8}{|l|}{$\mathrm{Fe}^{+9}$} \\
\hline \multirow[t]{5}{*}{$3 p^{5}$} & $1 s_{1 / 2}$ & 7444 & 7351 & 7362 & 7535 & 7195.87 & 7415.76 \\
\hline & $2 s_{1 / 2}$ & 1075 & 1100 & 1092 & 1129 & 1064.47 & 1107.25 \\
\hline & $2 p_{1 / 2}$ & 960 & 964 & 959 & 1013 & 944.59 & 995.98 \\
\hline & $2 p_{3 / 2}$ & 950 & - & - & 1000 & 915.62 & 966.05 \\
\hline & $3 s_{1 / 2}$ & 299 & 297 & 299 & 297 & 298.66 & 313.68 \\
\hline \multicolumn{8}{|l|}{$\mathrm{Fe}^{+12}$} \\
\hline \multirow[t]{5}{*}{$3 p^{2}$} & $1 s_{1 / 2}$ & 7564 & 7506 & 7506 & 7686 & 7320.14 & 7540.03 \\
\hline & $2 s_{1 / 2}$ & 1183 & 1221 & 1230 & 1234 & 1187.43 & 1230.29 \\
\hline & $2 p_{1 / 2}$ & 1070 & 1086 & 1093 & 1123 & 1055.50 & 1107.06 \\
\hline & $2 p_{3 / 2}$ & 1060 & - & - & 1110 & 1033.26 & 1083.98 \\
\hline & $3 s_{1 / 2}$ & 387 & 393 & 393 & 388 & 380.68 & 396.34 \\
\hline
\end{tabular}


Table 9

Comparison of transition energies (Ryd) from the ground state of Ni-like Xe computed using the NRSHM and Smith's SHM model, with experimental values. Relative errors $e_{\mathrm{r}}(\%)$ have been calculated for each model.

\begin{tabular}{llllll}
\hline & Observed & NRSHM & Smith & $e_{\mathrm{r}}^{\mathrm{NRSHM}}(\%)$ & $e_{\mathrm{r}}^{\text {Smith }}(\%)$ \\
\hline $3 d_{5 / 2} 4 s_{1 / 2}$ & 43.475 & 44.402 & 41.64 & -2.13 & 4.22 \\
$3 d_{3 / 2} 4 s_{1 / 2}$ & 44.448 & 45.178 & 42.62 & -1.64 & 4.11 \\
$3 d_{3 / 2} 4 p_{1 / 2}$ & 48.405 & 48.768 & 47.82 & -0.75 & 1.21 \\
$3 d_{5 / 2} 4 p_{3 / 2}$ & 48.817 & 49.360 & 47.58 & -1.11 & 2.53 \\
$3 d_{3 / 2} 4 p_{3 / 2}$ & 49.726 & 50.358 & 48.54 & -1.27 & 2.39 \\
$3 d_{5 / 2} 4 f_{7 / 2}$ & 62.339 & 63.250 & 61.44 & -1.46 & 1.44 \\
$3 d_{3 / 2} 4 f_{5 / 2}$ & 63.962 & 63.324 & 62.38 & 1.00 & 2.47 \\
\hline
\end{tabular}

quantities was already shown in Fig. 1. Nevertheless, in order to compare them with other model different to FAC, in Table 9 we show the transition energies for the ground state of Ni-like $\mathrm{Xe}$ compared with experimental data measured by Badnell et al. [47] obtained from the paper of Smith [22]. The NRSHM shows good
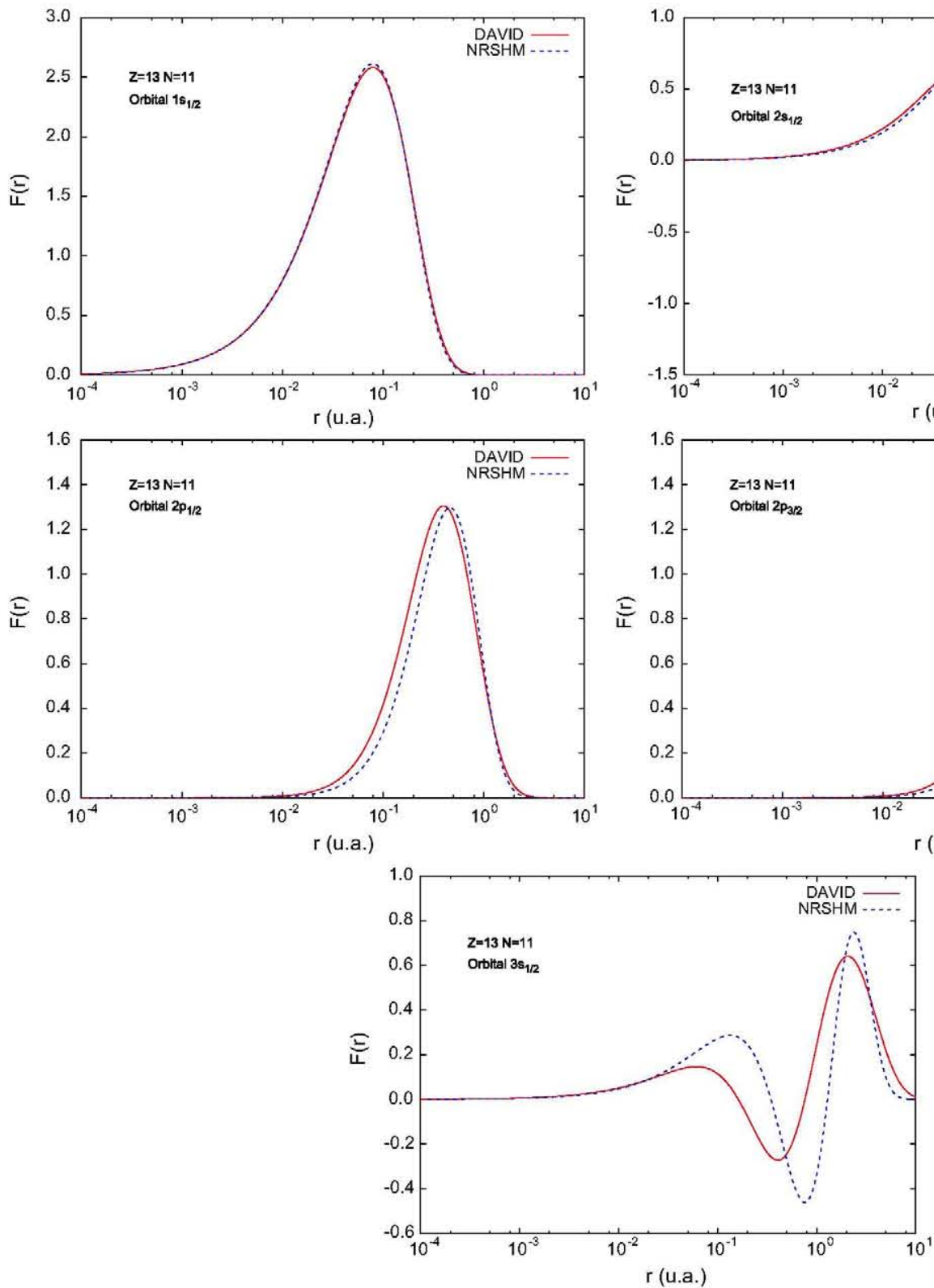

Fig. 4. Large component of the wave function for $1 s_{1 / 2}, 2 s_{1 / 2}, 2 p_{1 / 2}, 2 p_{3 / 2}$ and $3 s_{1 / 2}$ orbitals of a $\mathrm{Na}$-like $\mathrm{Al}$ ion. agreement with experimental data and with those obtained from Smith's model, having errors lower than $2.2 \%$.

\subsection{Wave functions}

One advantage of the SHM is that it provides analytical expressions for the wave functions $\psi_{n l j}$ depending on the screened charges. In this work we use the expressions of Dirac's radial wave functions proposed by Nikiforov et al. [23].

$F_{n l j}\left(r, Q_{n l j}\right)=\frac{B_{n l j}}{4 a v(K-\nu)} x^{\nu} e^{-\frac{x}{2}}\left[f_{1} x L_{n-j-\frac{3}{2}}^{2 v+1}(x)+f_{2} L_{n-j-\frac{1}{2}}^{2 \nu-1}(x)\right]$

$G_{n l j}\left(r, Q_{n l j}\right)=\frac{B_{n l j}}{4 a \nu(\kappa-\nu)} x^{\nu} e^{-\frac{\chi}{2}}\left[g_{1} x L_{n-j-\frac{3}{2}}^{2 \nu+1}(x)+g_{2} L_{n-j-\frac{1}{2}}^{2 \nu-1}(x)\right]$
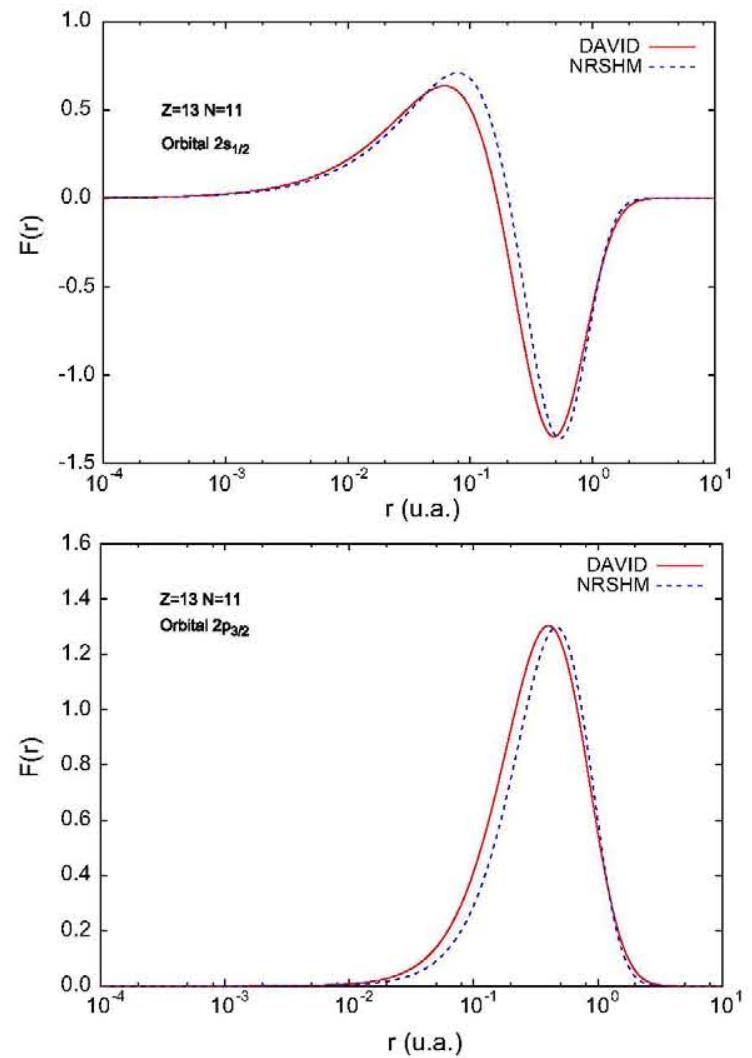

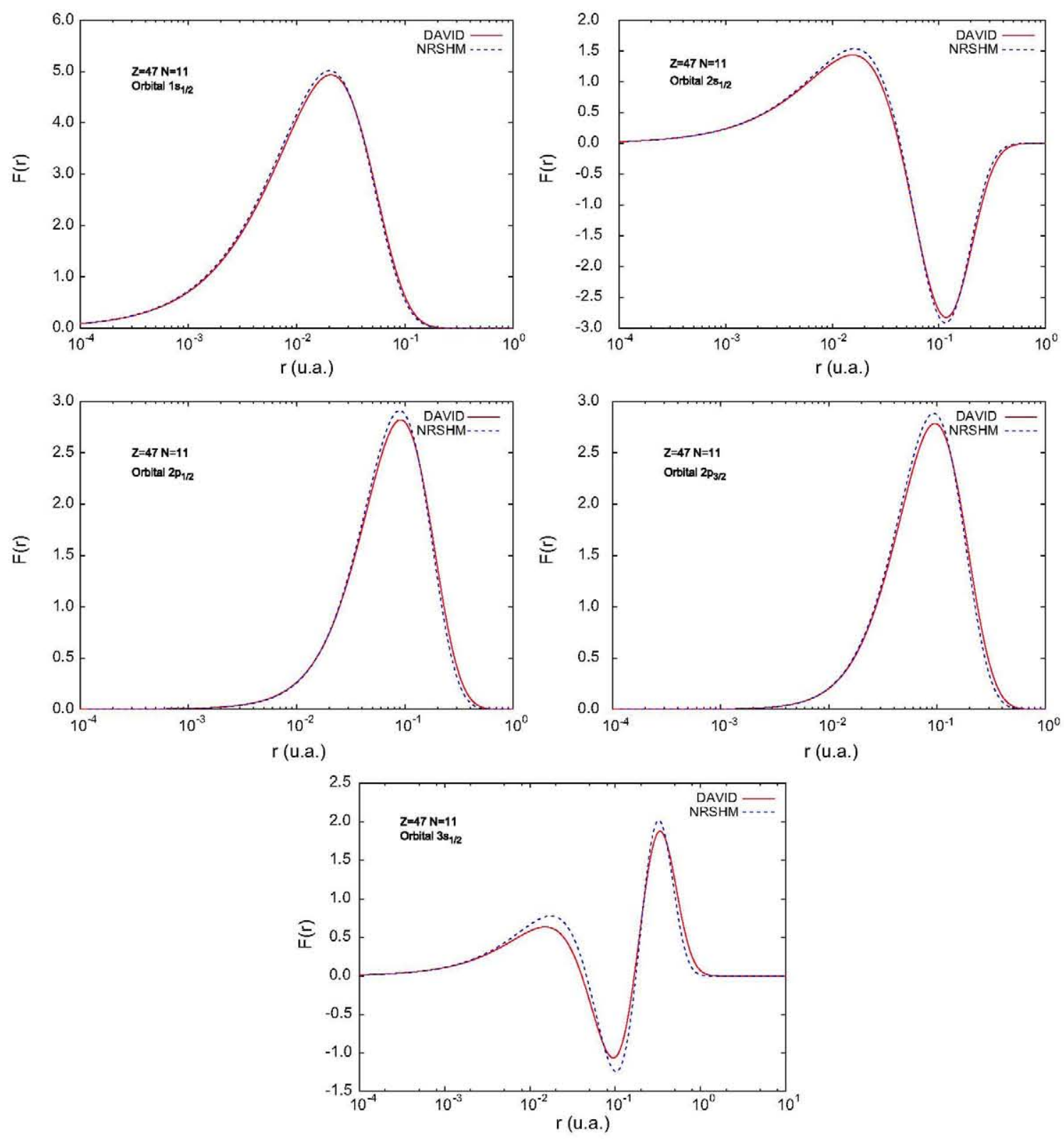

Fig. 5. Large component of the wave function for $1 s_{1 / 2}, 2 s_{1 / 2}, 2 p_{1 / 2}, 2 p_{3 / 2}$ and $3 s_{1 / 2}$ orbitals of a $\mathrm{Na}-$ like $\mathrm{Ag}$ ion.

where $F_{n l j}$ and $G_{n l j}$ are the large and small radial components of the wave function, respectively, expressed in term of the generalized Laguerre polynomials $L_{k}^{k \prime}(x)$ that depend on the variable $x=2 a c r$, where $c$ is the speed of light, $r$ the radial coordinate and $a$ a parameter depending on the screened charge, defined as:

$$
a=\frac{\xi}{\sqrt{\left(n-j-\frac{1}{2}+\nu\right)^{2}+\xi^{2}}}
$$

where $\xi=Q_{n l j} / c$ and $v=\sqrt{(j+1 / 2)^{2}-\xi^{2}}$. The rest of the terms in Eq. (10) are given by:

$$
\begin{aligned}
& \kappa=-2(j-l)\left(j+\frac{1}{2}\right), f_{1}=\frac{a \xi^{2}}{a \kappa\left(n-j-\frac{1}{2}+\nu\right)-\xi \nu}, f_{2}=\kappa-\nu \\
& g_{1}=\frac{f_{1} f_{2}}{\xi}, \quad g_{2}=\xi
\end{aligned}
$$

Finally, the normalization constant has the following expression:

$$
B_{n l j}=\frac{2 a^{2}}{\xi} \sqrt{\frac{f_{2}\left(n-j-\frac{1}{2}\right) !\left[a \kappa\left(n-j-\frac{1}{2}+\nu\right)-\xi \nu\right]}{\Gamma\left(n-j-\frac{1}{2}+2 \nu\right)}}
$$

Figs. 4 and 5 show large components of the radial wave function for $1 s_{1 / 2}, 2 s_{1 / 2}, 2 p_{1 / 2}, 2 p_{3 / 2}$ and $3 s_{1 / 2}$ orbitals of $\mathrm{Na}$-like $\mathrm{Al}$ and $\mathrm{Ag}$ ions, respectively. The screened hydrogenic wave functions have a reasonable behaviour for inner orbitals but get worse for outer orbitals, although again, the agreement improves as the ionization state increases, since the importance of the electron-nucleus interaction increase with increased ionization.

\section{Summary and conclusions}

We have computed a new set of universal screening constants in the context of an atomic relativistic model based on the Dirac's eigenvalue expression. A large energy level database has been used 
to fit the constants: it contains 61,356 values of ionization and excitation energies. The distribution of relative errors obtained is centered on the origin and has $88 \%$ of the errors deviations less than $\pm 10 \%$ relative to the values in the database. The model is competitive when comparing with more sophisticated codes and agrees with experimental data across its range of validity. The higher ionization state the better accuracy of the results. It also improves on the results of other screened hydrogenic models that do not include $j$-splitting. Future work will extend the set of relativistic coefficients beyond the $5 p_{3 / 2}$ level.

The model provides in a fast and simple way the atomic data needed to calculate the equation of state and radiative properties of plasmas under LTE or NLTE conditions with a reasonable accuracy. A preliminary version of the model has been integrated in the ATMED code $[48,49]$, an average atom code for computing opacities and the equation of state of LTE plasmas, obtaining good results in relation to other models presented in the literature.

\section{Acknowledgements}

This work has been partially supported by a Research Project of the Spanish Ministry of Science and Innovation (ENE2009-11208), by a Research Project of the "Agencia Canaria de Investigación, Innovación y Sociedad de la Información" of the regional government of the Canary Islands. (SOLSUBC2008000057) and also by the "Keep-in Touch" project of the European Union. The authors thankfully acknowledge the computer resources from Atlante, technical expertise and assistance provided by the Spanish Supercomputing Network (RES) and Instituto Tecnológico de Canarias Gobierno de Canarias.

\section{Appendix A. Relativistic expression for external screening}

In the $X \alpha$ model the electron binding energies are calculated by derivation of the total energy of an electronic configuration $E_{\mathrm{T}}$ with respect to the population $P_{k}$

$\epsilon_{k}=\frac{\partial E_{T}}{\partial P_{k}}$

where the expression for $E_{\mathrm{T}}$ is given by equation (1). Resolving we obtain for $\epsilon_{k}$ :

$\epsilon_{k}=\frac{\partial E_{T}}{\partial P_{k}}=\sum_{k^{\prime}} \frac{\partial}{\partial P_{k}}\left(P_{k^{\prime}} \cdot \epsilon_{k^{\prime}}\right)=\sum_{k^{\prime}}\left[\frac{\partial P_{k^{\prime}}}{\partial P_{k}} \cdot \epsilon_{k^{\prime}}+P_{k^{\prime}} \cdot \frac{\partial \epsilon_{k^{\prime}}}{\partial P_{k}}\right]$

$\epsilon_{k}=\frac{\partial E_{T}}{\partial P_{k}}=\epsilon_{k}+\sum_{k^{\prime}} P_{k^{\prime}} \frac{\partial \epsilon_{k^{\prime}}}{\partial Q_{k^{\prime}}} \frac{\partial Q_{k^{\prime}}}{\partial P_{k}}=\epsilon_{k}+A_{k}$

Therefore the electron binding energies are the sum of the Dirac's eigenvalue plus one second term $A_{k}$ corresponding to the external screening. In order to obtain the analitical expression for $A_{k}$ the two derivatives inside the summation have to be solved.

The derivative of the nuclear charge with respect to the populations is:

$$
\begin{aligned}
& \frac{\partial Q_{k^{\prime}}}{\partial P_{k}}=\frac{\partial}{\partial P_{k}}\left[Z-\sum_{j=1}^{j_{\max }} \sigma_{k^{\prime} j}\left(P_{j}-\delta_{k^{\prime} j}\right)\right]=-\sum_{j=1}^{j_{\max }} \sigma_{k^{\prime} j} \frac{\partial\left(P_{j}-\delta_{k^{\prime} j}\right)}{\partial P_{k}} \\
& \frac{\partial Q_{k^{\prime}}}{\partial P_{k}}=-\sigma_{k^{\prime} k} \quad \forall j=k
\end{aligned}
$$

To find the expression for the derivative of the Dirac's eigenvalue with respect to the nuclear charge, we introduce $a=n_{k^{\prime}}-j_{k^{\prime}}-1 / 2$ and $b=\left(j_{k^{\prime}}+1 / 2\right)^{2}$ in equation (2), and resolving the next formula is obtained:

$$
\begin{aligned}
\frac{\partial \epsilon_{k^{\prime}}}{\partial Q_{k^{\prime}}}= & -\frac{1}{\alpha^{2}}\left[1+\left(\frac{\alpha Q k^{\prime}}{a+\sqrt{b-\left(\alpha Q_{k^{\prime}}\right)^{2}}}\right)^{2}\right]^{-3 / 2} \\
& \times \frac{\alpha Q_{k^{\prime}}}{a+\sqrt{b-\left(\alpha Q_{k^{\prime}}\right)^{2}}} \cdot \frac{\alpha\left(a-\left(\alpha Q_{k^{\prime}}\right)^{2}+b\right)}{\left(a+\sqrt{b-\left(\alpha Q_{k^{\prime}}\right)^{2}}\right)^{2} \sqrt{b-\left(\alpha Q_{k^{\prime}}\right)^{2}}}
\end{aligned}
$$

Finally, the expression for the relativistic external screening is:

$$
\begin{aligned}
A_{k}= & \frac{1}{\alpha^{2}} \sum_{k^{\prime}} P k^{\prime} \sigma k^{\prime} k\left[1+\left(\frac{\alpha Q_{k^{\prime}}}{a+\sqrt{b-\left(\alpha Q_{k^{\prime}}\right)^{2}}}\right)^{2}\right]^{-3 / 2} \\
& \times \frac{\alpha Q_{k^{\prime}}}{a+\sqrt{b-\left(\alpha Q_{k^{\prime}}\right)^{2}}} \cdot \frac{\left.\alpha\left(a \sqrt{b-\left(\alpha Q_{k^{\prime}}\right.}\right)^{2}+b\right)}{\left(a+\sqrt{b-\left(\alpha Q_{k^{\prime}}\right)^{2}}\right)^{2} \sqrt{b-\left(\alpha Q_{k^{\prime}}\right)^{2}}}
\end{aligned}
$$

\section{Appendix B. Relativistic expression for HF electronic binding energies}

The relationship between $X \alpha$ and HF one electronic energies is given by Faussurier [19]:

$\epsilon^{\mathrm{HF}}=\epsilon^{\mathrm{X} \alpha}-\frac{\sigma_{k k}}{r_{k}}$

Here, $\sigma_{k k}$ is the screening constant for orbital $k$ and $r_{k}$ is the radius of the same orbital, which, in a relativistic approximation is calculated as [50]:

$r_{k}=\frac{1}{\left\langle\psi_{k}|1 / r| \psi_{k}\right\rangle}=\frac{\tilde{n} \sqrt{\tilde{n}^{2}+\alpha^{2} Q_{k}^{2}}}{Q_{k}}$

being:

$\tilde{n}=n_{k}-|\kappa|+|s| \kappa=-2\left(j_{k}-l_{k}\right)\left(j_{k}+\frac{1}{2}\right)$

$s=\sqrt{\kappa^{2}-\alpha^{2} Q_{k}^{2}}$

and where $n_{k} l_{k}$ and $j_{k}$ are the quantum numbers for orbital $k, Q_{k}$ is its screened charge and $\alpha$ is the fine structure constant.

\section{References}

[1] W.A. Lokke, W.H. Grassberger. XNQ. A Non-LTE Emission and Absorption Coefficient Subroutine, Technical Report, Report UCRL-5227, LLNL unpublished, 1977.

[2] G.D. Tsakiris, K. Eidmann, Journal of Quantitative Spectroscopy and Radiative Transfer 38 (1987) 353-368.

[3] K. Eidmann, Laser and Particle Beams 12 (1994) 223-244.

[4] S. Bel'kov, Journal of Experimental and Theoretical Physics (1997).

[5] G. Faussurier, Physical Review E 59 (1999) 7096-7109.

[6] G. Faussurier, C. Blancard, E. Berthier, Physical Review E 63 (2001) 026401.

[7] H.-K. Chung, M. Chen, W. Morgan, Y. Ralchenko, R. Lee, High Energy Density Physics 1 (2005) 3-12.

[8] H. Scott, S. Hansen, High Energy Density Physics 6 (2010) 39-47.

[9] S.A. Bel'kov, S.V. Bondarenko, E.I. Mitrofanov, Quantum Electronics 30 (2000) 963.

[10] G. Faussurier, C. Blancard, P. Renaudin, High Energy Density Physics 4 (2008) 114-123. 
[11] Q. Porcherot, G. Faussurier, C. Blancard, High Energy Density Physics 6 (2010) $76-83$.

[12] N. Gupta, B. Godwal, Laser and Particle Beams 19 (2001) 259-265.

[13] J.C. Slater, Physical Review 36 (1930) 57-64.

[14] H. Mayer, Methods of Opacity Calculations Technical Report, (Report LA-647) Los Alamos Scientific Laboratory, Los Alamos, NM, 1947.

[15] D. Layzer, Annals of Physics 8 (1959) 271-296.

[16] R. More, Journal of Quantative Spectroscopy \& Radiative Transfer 27 (1982) $345-357$.

[17] F. Perrot, Physica Scripta 39 (1989) 332

[18] R. Marchand, S. Caill, Y.T. Lee, Journal of Quantitative Spectroscopy and Radiative Transfer 43 (1990) 149-154.

[19] G. Faussurier, C. Blancard, A. Decoster, Journal of Quantitative Spectroscopy and Radiative Transfer 58 (1997) 233-260.

[20] S. Kawata, S. Kato, S. Kiyokawa, Japanese Journal of Applied Physics 32 (1993) 5681-5691

[21] J. Pomarico, D.I. Iriarte, H.O.D. Rocco, Brazilian Journal of Physics 35 (2005) $530-535$.

[22] C.C. Smith, A screened hydrogenic model with fine structure splitting. High Energy Density Physics 7 (1) (2011) 1-5. doi:10.1016/j.hedp.2010.11.001.

[23] A. Nikiforov, V. Novikov, A.D. Solomyannaya, Laser and Particle Beams 14 (4) (1996a) 765-779.

[24] A. Nikiforov, V. Novikov, A.D. Solomyannaya, High Temperature 34 (1996b) $220-233$.

[25] J.G. Rubiano, R. Rodriguez, J.M. Gil, F.H. Ruano, P. Martel, E. Minguez, Journal of Quantitative Spectroscopy and Radiative Transfer 72 (2002) 575-588.

[26] J.G. Rubiano, R. Florido, R. Rodriguez, J.M. Gil, P. Martel, E. Minguez, Journal of Quantitative Spectroscopy and Radiative Transfer 83 (2004) 159-182.

[27] P. Martel, L. Doreste, E. Mnguez, J.M. Gil, Journal of Quantitative Spectroscopy and Radiative Transfer 54 (1995) 621-636.

[28] P. Martel, J. Rubiano, J. Gil, L. Doreste, E. Mnguez, Journal of Quantitative Spectroscopy and Radiative Transfer 60 (1998) 623-633.

[29] Y. Ralchenko, F.-C. Jou, D. Kelleher, A. Kramida, A. Musgrove, J. Reader, W. Wiese, K. Olsen, National Institute of Standards and Technology, Gaithersburg, MD, 2007.

[30] M.F. Gu, AIP Conference Proceedings 730 (2004) 127-136

[31] D.A. Coley, An Introduction to Genetic Algorithms for Scientists and Engineers World Scientific Publishing, 1999.
[32] J.R. Koza. Genetic Programming: On the Programming of Computers by Means of Natural Selection (Complex Adaptive Systems), 1992.

[33] A. Novobilski, F.A. Kamangar, Absolute Percent Error Based Fitness Functions for Evolving Forecast Models. AAAI Press, 2001.

[34] K. Deb, Computer Methods in Applied Mechanics and Engineering 186 (2000) 311-338.

[35] E. Moore, Atomic energy levels, National Bureau of Standards, NRSDS-NBS 35 (1971).

[36] C.J. Roothaan, P.S. Kelly, Physical Review 131 (1963) 1177-1182.

[37] P.S. Kelly, Journal of Quantative Spectroscopy \& Radiative Transfer 4 (1964) 117

[38] A. Libermann, A. Zangwill, Computer Physics Communications 32 (1984) 75-82

[39] S. Fraga, Karwowski, M. Saxena, Handbook of Atomic Data. Elsevier, New York 1976.

[40] J.C. Slater, Physical Review 34 (1929) 1293-1322.

[41] P. Jonsson, X. He, C.F. Fischer, I. Grant, Computer Physics Communications 177 (2007) 597-622.

[42] L.G. Dyachkov, P.M. Pankratov, Journal of Physics B: Atomic, Molecular and Optical Physics 28 (1995) 1173.

[43] T. Yabe, B. Goel, Kernforschungzentrum karlsruhe (1986) Report No. KfK-4176 (Karlsruhe).

[44] S. Mabong, G. Maynard, K. Katsonis, Laser and Particle Beams 14 (1996) $575-586$.

[45] J. Rogers, B.G. Wilson, C.A. Iglesias, Physical Review A 38 (1988) 5007-5020.

[46] W. Lotz, Journal of Optical Society of America 58 (1968) 915-916.

[47] N.R. Badnell, K.A. Berrington, H.P. Summers, M.G. O'Mullane, A.D. Whiteford, C.P. Ballance, Journal of Physics B: Atomic, Molecular and Optical Physics 37 (2004) 4589.

[48] J.G. Rubiano, M.A. Mendoza, J.M. Gil, R. Rodriguez, R. Florido, P. Martel, E. Minguez. In: 37th EPS Conference on Plasma Physic., volume $34 \mathrm{~A}$ of Europhysics conference abstracts, organization European Physics Society, p. P5.405.

[49] M.A. Mendoza, J.G. Rubiano, J.M. Gil, R. Rodriguez, R. Florido, P. Martel, E. Minguez. In: 37th EPS Conference on Plasma Physic., volume $34 \mathrm{~A}$ of Europhysics conference abstracts, organization European Physics Society, p. P4.410.

[50] J. Kobus, J. Karwowski, W. Jaskolski, Journal of Physics A: Mathematical and General 20 (1987) 3347. 